\title{
Analysis of defects on BN nano-structures using high-resolution electron microscopy and density-functional calculations
}

\author{
Erman Bengu $^{\mathrm{a}, *}$, Laurence D. Marks ${ }^{\mathrm{b}}$, Rasim V. Ovali ${ }^{\mathrm{c}}$, Oguz Gulseren ${ }^{\mathrm{c}}$ \\ a Department of Chemistry, Institute of Materials Science and Nanotechnology, Bilkent University, Ankara, Turkey \\ ${ }^{\mathrm{b}}$ Department of Materials Science and Engineering, Northwestern University, Evanston, IL 60208, USA \\ c Department of Physics, Bilkent University, Ankara, Turkey
}

\section{A R T I C L E I N F O}

PACS:

68.37.Lpd

81.05.Tp

81.15.Aa

71.15.Nc

Keywords:

Microscopy of surfaces

Interfaces and thin films

Fullerenes and related materials

Models of film growth

Total energy and cohesive energy

calculations

\begin{abstract}
A B S T R A C T
Cubic boron nitride (c-BN) nucleation takes place on hexagonal boron nitride (h-BN) layers growing perpendicular to the substrate surface during thin film synthesis. Studies focused on the nucleation of the cubic phase suggest the possibility that transient phases and/or defects on these h-BN structures have a role in sp3-bonded cubic phase nucleation. In this study, we have investigated the nature, energetics, and structure of several possible defects on BN basal planes, including point defects, 4-, and 5-fold BN rings, that may possibly match the experimentally observed transient phase fine structure. TEM image observations are used to build approximate atomic models for the proposed structures, and DFT calculations are used to relax these structures while minimizing their respective total energies. These optimized atomic geometries are then used to simulate TEM images, which are compared to the experimentally observed structures. Data from DFT calculations and analysis of simulated images from the proposed atomic structures suggest that 4-fold BN rings are more likely to exist on the transient phase possibly leading to c-BN nucleation.
\end{abstract}

(c) 2008 Elsevier B.V. All rights reserved.

\section{Introduction}

One of the earliest articles reporting synthesis of diamond from graphite using a high temperature and pressure technique has been published by Bundy et al. [1]. A similar method has also been followed by Bundy and Wentorf [2] while synthesizing c-BN from its hexagonal polymorph. Hardness of c-BN has been found to reach up to $70 \mathrm{GPa}$; thermal conductivity is reported to be between 2 and $13 \mathrm{~W} \mathrm{~cm}^{-1} \mathrm{~K}^{-1}$; the band gap is approximately $6.4 \mathrm{eV}$ [3]. In thin film form, c-BN has been first deposited by Sokolowski et al. [4]. Since then, there have been a large number of studies on the deposition of c-BN; however, synthesis of thick, high-quality c-BN films remains a significant challenge.

Nucleation and growth of the cubic phase have also drawn significant interest due to its complexity and unusual structure. Cross-sectional TEM (XTEM) studies on c-BN thin films have indicated that the c-BN phase always forms on top of an h-BN layer where the basal planes of h-BN grow perpendicular to the substrate surface. Bombardment of the growing film with ions has also been considered essential for the stabilization of the cubic phase. There have been a number of models proposed for explaining the mechanisms involved in c-BN thin film nucleation

\footnotetext{
* Corresponding author. Tel.: +903122902153.

E-mail address: bengu@fen.bilkent.edu.tr (E. Bengu).
}

and growth. Refs. [5,6] contain comparative reviews for these models. Most discussed models include the compressive stress model [7-9], preferential sputtering model [10,11], sub-plantation model [12-15], thermal spike model [16-18], and the nanoarches model [5]. It has been observed that, after the cubic phase nucleates on top of the h-BN basal planes, the energy of the ions could be lowered significantly while the growth of the cubic phase is maintained [19]. Therefore, the h-BN to c-BN transformation can be classified as nucleation-limited, following the classical nucleation theory nomenclature.

The nanoarch model, unlike others, proposes arches as $a$ transient structure on the h-BN layer triggered by intense ballistic displacements due to ion- and/or electron-beam bombardment of the growing h-BN film [20]. This model explains the necessity of the h-BN layer preceding the cubic phase as observed in XTEM. Fig. 1(a) displays TEM images of the edge of an h-BN particulate after intense electron-beam bombardment inside a Hitachi UHV H-9000 TEM, Fig. 1(b) shows arches on a growing h-BN film, and Fig. 1(c) depicts a basic atomic model of a two-layer nanoarch. These arches, according to the model, act as a preferential site for the sp3 phase nucleation at the boundary between h-BN and c-BN. In a way, these arches can be considered as a surface reconstruction where, given sufficient activation energy, atoms at the termination edges of the $\mathrm{BN}$ basal planes can rearrange themselves to minimize the number of unsatisfied bonds. The radius of curvature affects the bonding scheme within the $\mathrm{BN}$ 

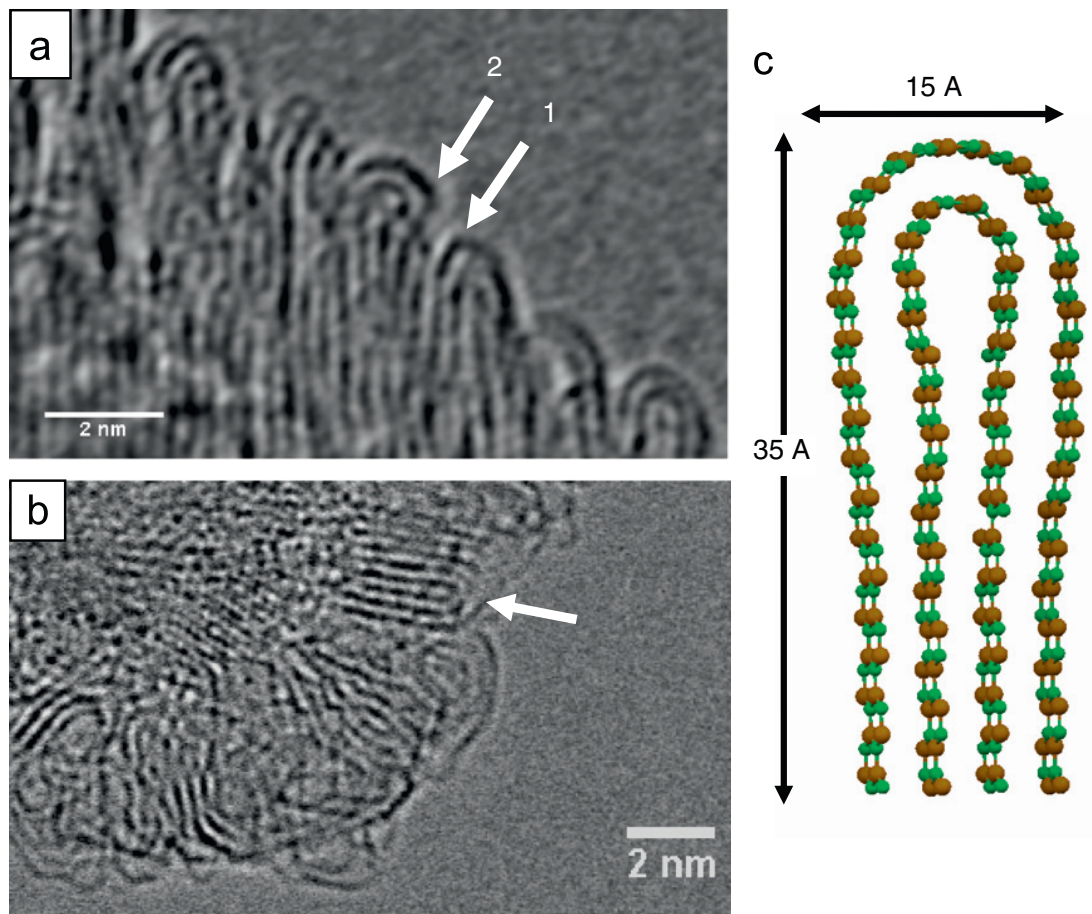

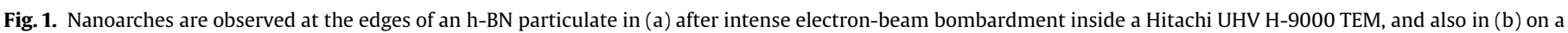
growing h-BN film (fine lattice fringes are due to well-ordered h-BN viewed along ( 0001$)$ zone). A basic atomic model of a two-layer nanoarch is shown in (c).

sheet at the tip of the arch, which is otherwise near-planar $\mathrm{sp}^{2}$ bonding observed in $\mathrm{BN}$ sheets for $\mathrm{h}-\mathrm{BN}$. While the nanoarches enable the total energy of the system to be minimized, the new arrangement of atoms on the surface may act as nucleation sites for c-BN growth.

In this study, our purpose is to investigate the local atomic arrangements on the nanoarches to provide sensible clues for a better understanding of the phase transition from $\mathrm{sp2}$ - to $\mathrm{sp} 3$ bonded $\mathrm{BN}$. Unique formations on the nanoarches are observed and investigated further; these may serve as the preferential sites for sp3 nucleation. Reasonable atomic models are built, matching the size and shape of the formations on arches. Relative stability of the candidate structures with respect to each other is also studied using total energy calculations through the first principles. Finally, TEM image simulations of structures, which are energetically favorable, are produced, and these are compared to experimentally observed ones. Our ultimate aim is to propose an atomic configuration for the observed structure that replicates the general configuration rather than to provide an accurate quantitative fit.

\section{Observation of fine structures on $B N$ nanoarches}

BN nanoarches via electron-beam route were synthesized on sub-micron-sized h-BN particles ground in methanol from the bulk, and the resulting suspension of fine particles were dispersed on a gold TEM grid. Details of these TEM sample preparation and electron-bombardment experiments were provided in Refs. $[5,20]$. BN thin films with the confirmed presence of nanoarches were also deposited with a unique ion-beam-assisted deposition system attached to a UHV surface analysis and characterization system [21]. Further details of this experimental setup and c-BN deposition can be found in Refs. [22,23].

Although it is not strikingly obvious at first glance, detailed analysis of the TEM micrograph in Fig. 1(a) evidences sharp corners, disclinations, on the arches, which suggest the underlying atomic configuration is very likely to be different than the atomic model depicted in Fig. 1(c), which is a semi-circular arrangement. The first of these disclinations is close to the peak of the arch marked " 1 ", and another one is observed on the right side of the arch labelled as "2".

\section{Structure and energetics of possible defects in BN nanoarches}

There have been a number of theoretical studies of the energetics of zero-dimensional defects (i.e. vacancies, anti-site defects) for BN monolayers [24], while direct observation of such atomic level defects has been limited for BN nano-structures $[21,25]$. One possibility is that the disclinations observed on the arches could be induced by zero-dimensional defects. Such defects could be easily formed by electron-beam irradiation of h-BN particles in a TEM or on the growing film during c-BN synthesis via ion-beam bombardment [21,23].

Distortions induced on the BN basal planes by these defects are studied using a $(4,4) \mathrm{BN}$ basal plane shown in Fig. 2. We start our analysis by comparing the disclinations observed experimentally to the energy minimized geometry of the BN basal plane due to nitrogen vacancy $\left(V_{\mathrm{N}}\right)$, boron vacancy $\left(V_{\mathrm{B}}\right)$, boron anti-site $\left(B_{\mathrm{N}}\right)$, and nitrogen anti-site $\left(N_{\mathrm{B}}\right)$. Fig. 2 depicts the distortions in the atomic structure after energy relaxation of the BN basal planes due to the aforementioned point defects. Total energies for these structures were calculated from the first principles by the projector-augmented-wave (PAW) method using Vienna Ab-initio Simulation Package (VASP) program [26-30]. The exchange-correlation potential was expressed in terms of the generalized gradient approximation (GGA) (Perdew-Wang 91 type [31]). To achieve the desired accuracy, a plane-wave cutoff energy of $500 \mathrm{eV}$ was used in all calculations. For the periodic systems, we used a $1 \times 7 \times 1$ Monkhorst-Pack [32] mesh in order to model the $k$-point sampling in the Brillouin Zone. Ten angstrom of vacuum was used to minimize the ion-ion 


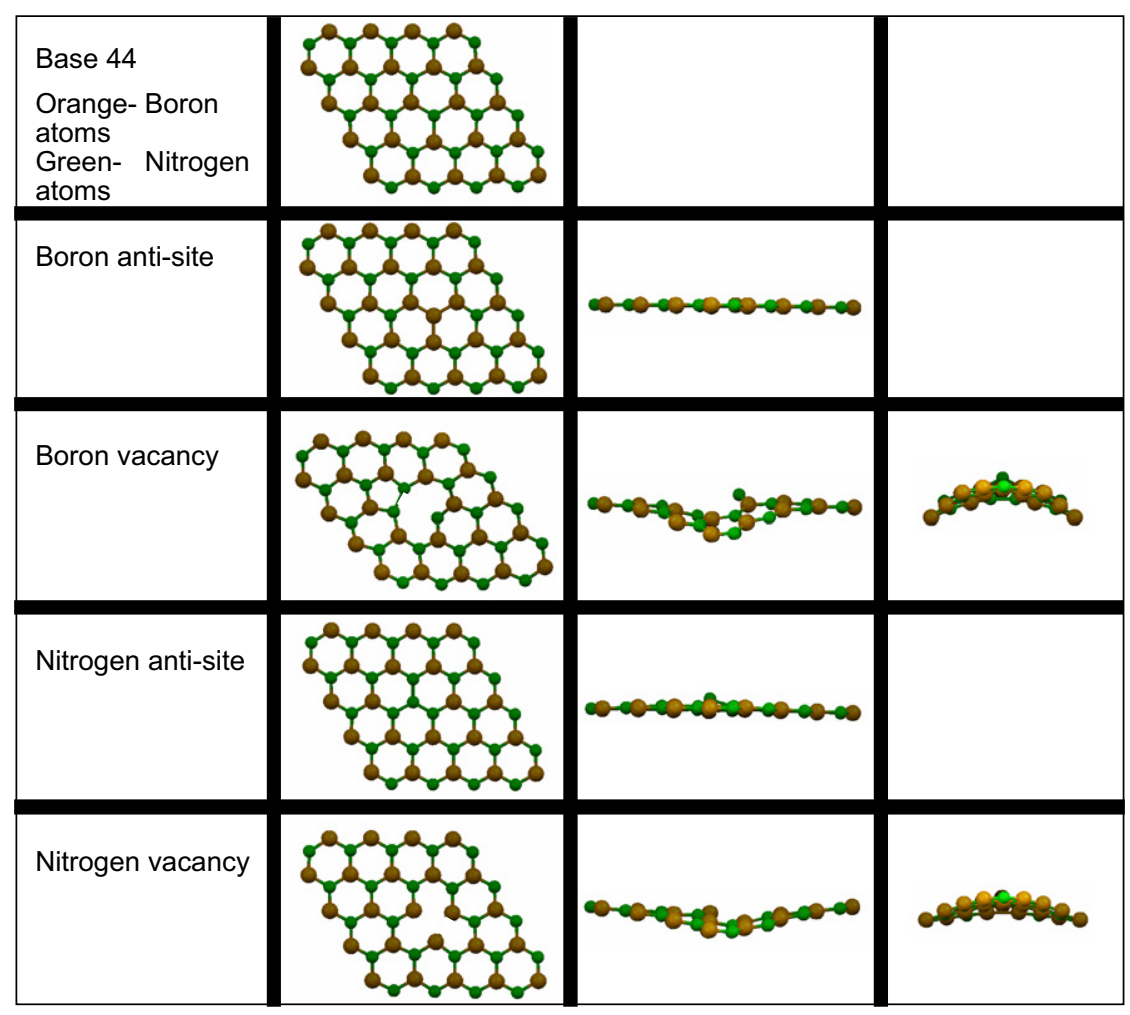

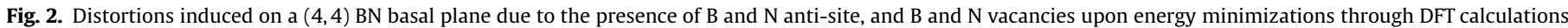
are depicted. While anti-site type point defects induced very small, localized distortions, vacancy defects did cause large, two-dimensional strain fields.

interaction in the non-periodic direction. The partial occupancy around the Fermi level is treated by Gaussian smearing with a smearing parameter of $0.08 \mathrm{eV}$. For all calculations energy was converged to within $10^{-4}-\mathrm{eV}$ accuracy.

Atomic configurations of the defects resulting from total energy minimizations were then used to simulate HREM images using a multislice algorithm [33] for comparison with observed images of the disclination on the BN nanoarch in Fig. 1(a). The image of the disclination and the simulated images from the defect structures are comparatively displayed in Fig. 3. Measurements indicate that the disclination for the experimentally observed nanoarch marked " 1 " is around $\sim 104^{\circ}$ and $\sim 102^{\circ}$ for "2", as shown in Fig. 3(a). The sharp corner observed for the external profile of the arch does not match any profiles of the simulated images for the zero-dimensional defects considered, as shown in Figs. 3(b)-(g).

An earlier study by Loiseau et al. [34] on the structure of BN nano-structures suggested the cap structure to be composed of a cone with a 4 -fold ring at the apex generating a $120^{\circ}$ disclination. Other studies, focused on the stability of BN fullerenes and nanotubes [35-37], verified the stability of BN structures with square rings (4-fold) over pentagon (5-fold) rings. Also, sharp angular profiles for BN nano-structures similar to those observed on the arches were replicated with the use of 4- and 8-fold rings by Bengu and Marks [21]. However, there have been other reports suggesting the stability of BN nano-structures with pentagonal rings, provided that synthesis conditions are either significantly B- or N-rich [38-40]. Such environments may exist under conditions used for BN thin film synthesis.

To investigate the possibility of higher order defects causing such distortions on the BN basal planes matching those observed on nanoarches, we prepared BN basal plane configuration with 4- and 5-fold ring defects. Special care was given to prepare defective $\mathrm{BN}$ structures with the same number of $\mathrm{B}$ and $\mathrm{N}$ atoms each. One reason for carefully matching the number of B-N pairs in our test structures was to ensure accurate determination of the formation energy per pair for these new set of defects. Fig. 4 shows the configuration of BN basal planes with 4-fold rings and 5 -fold rings after the geometries are relaxed for minimum total energy using the first principle methods described above. There are two types of 5-fold rings, one with a B-B pair and one with an N-N pair; both the atomic configurations are displayed in Fig. 4. Again using a multislice algorithm, HREM images of the structures were generated using two different imaging orientations. Although a large number of incident beam versus sample structure orientation combinations for simulations were considered during this study, only two are presented here as many produced disclinations with similar angles. Disclination angles ranged from $90^{\circ}$ to roughly $100^{\circ}$ for all three atomic configurations considered.

Atomic structures for the 4- and 5-fold structures when viewed in the two selected directions are shown in Figs. 4(a), (d), and (g) for orientation 1 and (b), (e), and (h) for orientation 2. Appropriate statistical noise was also added to the simulated images for completeness. We found that 5-fold N-pair and B-pair structures, under the simulation conditions used, resulted in arguably identical images. Therefore, we only included images generated from the B-pair atomic configuration for the two orientations considered, as shown in Figs. 4(f) and (i) for orientations 1 and 2, respectively. Also, simulated images from the 4-fold structures depicted in Figs. 4(a) and (b) for the two different viewing orientations were indistinguishable.

Comparative analyses of the simulated images versus the observed arch defect structures show reasonable qualitative match between the calculated and observed images for some of the sample tilts, as shown in Figs. 4(c), (f), and (i). Images from the 4-fold ring (Figs. 4(c)) and 5-fold ring (Fig. 4(f)) indicated a disclination angle and a sharp apex matching observations in 


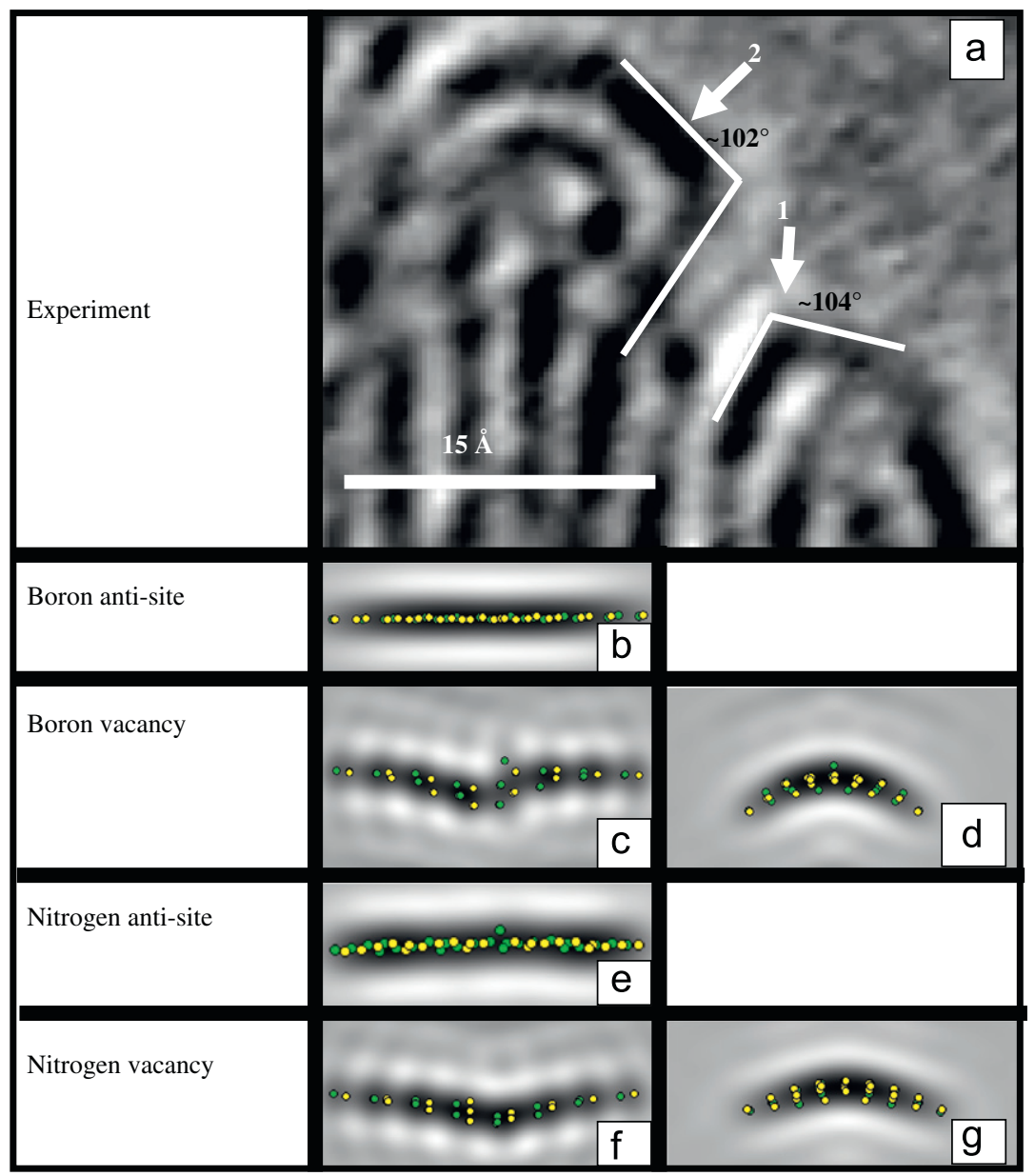

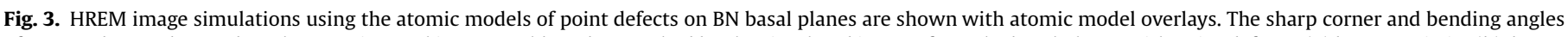

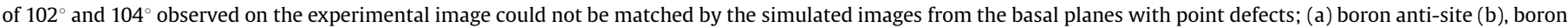
vacancy (c) and (d), nitrogen anti-site (e), nitrogen vacancy (f) and (g).

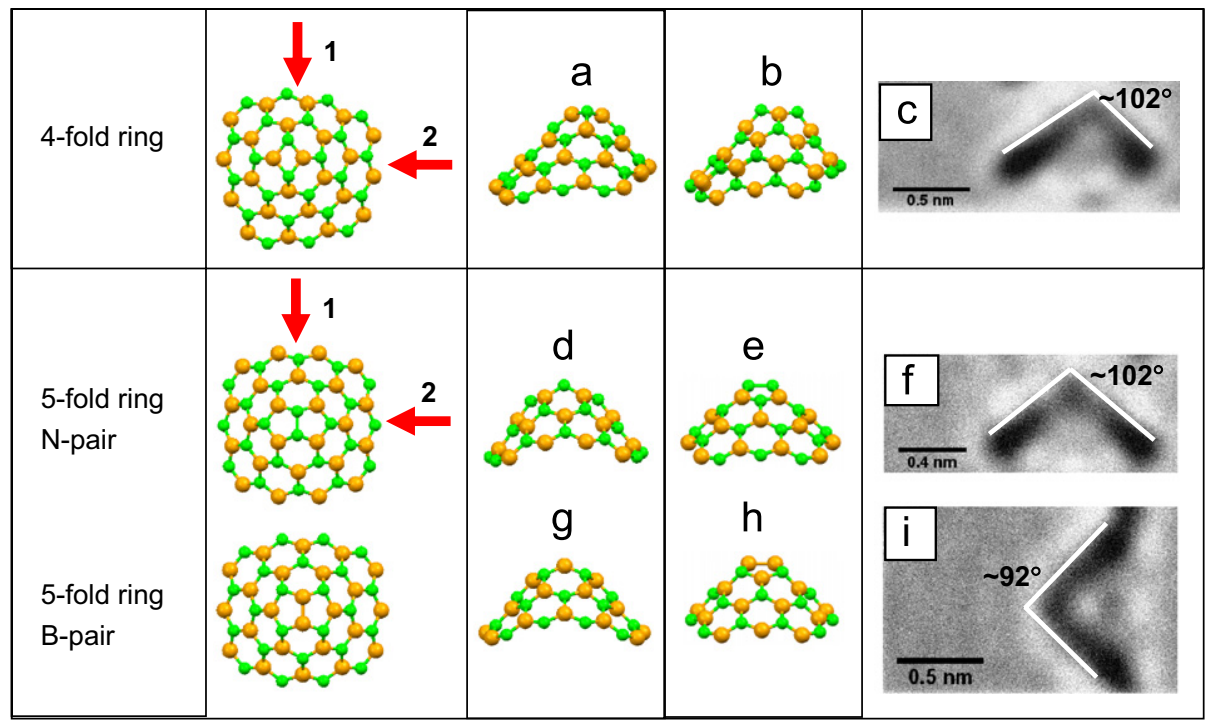

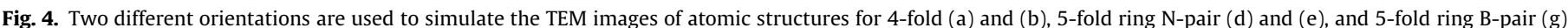

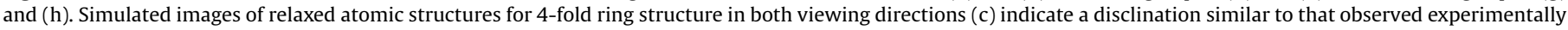

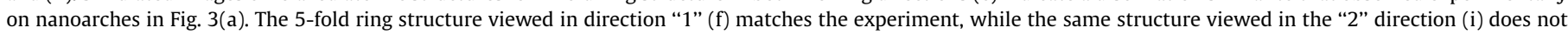
match the experiment.

Fig. 3(a). However, it should be noted that it is very difficult to make clear and quantitative decisions from these experimental images as to what should be the fine atomic structure, but complimentary methods do exist, which can help us take this analyses further, despite the limited resolution of these experimental images. 
Table 1

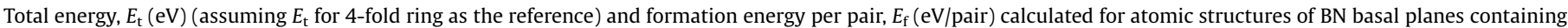
4- and 5-fold defects in B-rich or N-rich environments

\begin{tabular}{|c|c|c|c|c|}
\hline Type & Total number of atoms & Total energy, $E_{\mathrm{t}}$ & $E_{\mathrm{f}}, \mathrm{B}$-rich environment & $E_{\mathrm{f}}, \mathrm{N}$-rich environment \\
\hline 4-fold ring & B $24, \mathrm{~N} 24, \mathrm{H} 18$ & 0 & 0.391 & 0.391 \\
\hline 5-fold B-pair & В $24, \mathrm{~N} 24, \mathrm{H} 18$ & 0.6 & 0.416 & 0.416 \\
\hline 5-fold N-pair & B $24, \mathrm{~N} 24, \mathrm{H} 18$ & 1.18 & 0.440 & 0.440 \\
\hline
\end{tabular}

A better assessment with respect to which of these higher order defects might be more likely to exist upon electron-beam irradiation can be made using the energy of formation of defects per atom calculated through the first principles. Here, we define the formation energy as follows:

$E_{\text {form }}=E_{\text {tot }}-n_{\mathrm{N}} \mu_{\mathrm{N}}-n_{\mathrm{B}} \mu_{\mathrm{B}}-n_{\mathrm{H}} \mu_{\mathrm{H}}$

where $E_{\text {tot }}$ is the total ground state energy, $n$ 's are the number of atoms, and $\mu$ 's are the chemical potentials. Metallic alpha-boron phase for $\mathrm{B}$ and gas phases for $\mathrm{N}$ and $\mathrm{H}$ were used to calculate chemical potentials. Also for further completeness, energy calculations are adjusted to include consideration for a B- or $\mathrm{N}$-rich synthesis environment. We define the formation energy for $\mathrm{N}$-rich and B-rich environments by introducing a constraint on chemical potential by the equilibrium thermodynamic condition:

$\mu_{\mathrm{N}}+\mu_{\mathrm{B}}=\mu_{\mathrm{BN}}^{\text {layer }}$

where $\mu_{\mathrm{BN}}^{\text {layer }}$ is the chemical potential of a BN pair in an infinite planar boron nitride sheet. Hence, the formation energy becomes

$E_{\text {form }}^{\mathrm{N}-\text { rich }}=E_{\text {tot }}-n_{\mathrm{N}} \mu_{\mathrm{N}}-n_{\mathrm{B}}\left(\mu_{\mathrm{BN}}^{\text {layer }}-\mu_{\mathrm{N}}\right)-n_{\mathrm{H}} \mu_{\mathrm{H}}$

$E_{\text {form }}^{\mathrm{B}-\text { rich }}=E_{\text {tot }}-n_{\mathrm{N}}\left(\mu_{\mathrm{BN}}^{\text {layer }}-\mu_{\mathrm{B}}\right)-n_{\mathrm{B}} \mu_{\mathrm{B}}-n_{\mathrm{H}} \mu_{\mathrm{H}}$

We show the calculated formation energies of 4- and 5-fold ring defects in N-rich and B-rich environments in Table 1 . The resulting formation energy values indicate that the stability of a single 4-ring structure is higher than the other two structures with 5-ring configurations for both of the environmental conditions considered.

\section{Discussion}

The data from total energy calculations indicate that the energy of the structure with the 4-fold ring is less than that for the 5 -fold rings by $1.18 \mathrm{eV}$ for the ring with the $\mathrm{N}$-pair and $0.6 \mathrm{eV}$ with the B-pair for atomic structures using $24 \mathrm{~B}-\mathrm{N}$ pairs. The chemical activity of the environment with regard to boron or nitrogen does not seem to improve the energetics in favor of the 5-fold rings. While this finding at the first glance seems contradictory to what has been reported in earlier studies by Alexandre et al. [39] and Fowler et al. [40], in those studies the structures considered were non-stoichiometric fullerenes of $\mathrm{BN}$. In this study, we have investigated the fine atomic structure of nanoarches, which are similar to large diameter nanotubes with a cap. Hence, we believe that these structures are very likely to be stoichiometric over large areas barring local instabilities due to point defects.

Simulated images for the relaxed structures due to 4- or 5-fold defects both displayed qualitative resemblance to the observed structure, but clear cut evidence to support one over the other is not available through the analysis of these images. Furthermore, varying the sample tilt liberally, it is also possible to generate a range of similar disclination angles from various other defects that may exist on BN basal planes [41], but we have kept our analyses restricted to 4- and 5-fold rings as earlier theoretical studies suggested these to be more stable than others. Naturally, a microscope with corrected electron optics can provide better direct evidence for the local atomic structure of the BN nanoarches. However, it should be noted that the sample preparation is also a key factor for producing experimentally interpretable structure images. Keeping the number of atoms limited in our atomic models, we were able to take advantage of DFT calculations to decide which model was more likely to exist on the $\mathrm{BN}$ arches using relatively low-resolution data.

\section{Conclusion}

In this study, we have used a combined method comprising HREM imaging and DFT calculations to investigate and uncover the fine atomic structures observed for BN nanoarches. While image simulations of the BN basal plane geometries due to point defects after relaxation did not bear strong resemblance to that experimentally observed, defect configurations including 4- or 5 -fold rings did provide a qualitative match to the experiment. Furthermore, total energy calculations suggest that the 4-fold ring defect is energetically more favorable than its 5-fold counterpart under both B- and N-rich environments. It is possible that the 4 -fold rings are a candidate site for the ensuing c-BN nucleation during thin film growth.

\section{Acknowledgements}

We would like to acknowledge the support of the State Planning Agency, Bilkent University, National Nanotechnology Research Center project, for allowing the use of their computer facilities.

\section{References}

[1] F.P. Bundy, H.T. Hall, R.H. Wentorf, J. Chem. Phys. 38 (1955) 1114.

[2] F.P. Bundy, R.H. Wentorf, J. Chem. Phys. 38 (1963) 1144.

[3] L. Vel, G. Demazeau, J. Etourneau, Mater. Sci. Eng. B 10 (1991) 49.

[4] M. Sokolowski, A. Sokolowska, A. Rusek, Z. Romanowski, B. Gokieli, M. Gajewska, J. Cryst. Growth 52 (1981) 165

[5] C.C. Davila, E. Bengu, L.D. Marks, M. Kirk, Diamond Relat. Mater. 8 (1999) 1091

[6] P.B. Mirkarimi, K.F. McCarty, D.L. Medlin, Mater. Sci. Eng. Rep. 21 (1997) 47.

[7] D.R. Mckenzie, W.D. Mcfall, W.G. Sainy, C.A. Davis, R.E. Collins, Diamond Relat Mater. 2 (1993) 970.

[8] D.R. Mckenzie, D.H. Cockayne, D.A. Muller, M. Murakawa, S. Miyake, S. Watanabe, P. Fallon, J. Appl. Phys. 70 (1991) 3007.

[9] D.B. Shtansky, Y.Y. Takamura, T. Yoshida, Y. Ikuhara, Sci. Technol. Adv. Mater. 1 (2000) 219.

[10] W. Kulisch, S. Reinke, Diamond Films Technol. 7 (1997) 105.

[11] S. Reinke, M. Kuhr, W. Kulisch, Diamond Relat. Mater. 4 (1995) 272.

[12] S. Uhlmann, T. Frauenheim, U. Stephan, Phys. Rev. B 51 (1995) 3287.

[13] Y. Lifshitz, S.R. Kasi, J.W. Rabalais, W. Eckstein, Phys. Rev. B 41 (1990) 10468

[14] W. Dworschak, K. Jung, H. Ehrhardt, Thin Solid Films 254 (1995) 65.

[15] J. Robertson, J. Gerber, S. Sattel, M. Weiler, K. Jung, H. Ehrhardt, Appl. Phys Lett. 66 (1995) 3287.

[16] H. Feldermann, R. Merk, H. Hofsass, C. Ronning, T. Zheleva, Appl. Phys. Lett. 74 (1999) 1552.

[17] E. Franke, M. Schubert, J.A. Woollam, J.D. Hecht, G. Wagner, H. Neumann, F. Bigl, J. Appl. Phys. 87 (2000) 2593.

[18] H. Hofsass, H. Feldermann, R. Merk, M. Sebastian, C. Ronning, Appl. Phys. A 66 (1998) 153.

[19] P.B. Mirkarimi, D.L. Medlin, K.F. McCarty, D.C. Dibble, W.M. Clift, J.A. Knapp J.C. Barbour, J. Appl. Phys. 82 (1997) 1617. 
[20] C. Collazo-Davila, E. Bengu, C. Leslie, L.D. Marks, Appl. Phys. Lett. 72 (1998) 314.

[21] E. Bengu, L.D. Marks, Phys. Rev. Lett. 86 (2001) 2385.

[22] E. Bengu, C. Collazo-Davila, D. Grozea, E. Landree, I. Widlow, M. Guruz, L.D. Marks, Microsc. Res. Tech. 42 (1998) 295.

[23] C. Collazo-Davila, E. Landree, D. Grozea, G. Jayaram, R. Plass, P.C. Stair, L.D. Marks, J. Microsc. Soc. Am. 1 (1995) 267.

[24] Sergio Azevedo, J.R. Kaschny, Caio M.C. de Castilho, F. de Brito Mota, Nanotechnology 18 (2007) 495707.

[25] A. Zobelli, C.P. Ewels, A. Gloter, G. Seifert, O. Stephan, S. Csillag, C. Colliex, Nano Lett. 6 (2006) 1955.

[26] G. Kresse, J. Hafner, Phys. Rev. B 48 (1993) 13115.

[27] G. Kresse, J. Furthmüller, Comput. Mater. Sci. 6 (1996) 15

[28] G. Kresse, J. Furthmüller, Phys. Rev. B 54 (1996) 11169.

[29] G. Kresse, D. Joubert, Phys. Rev. B 59 (1999) 1758.

[30] P.E. Blöchl, Phys. Rev. B 50 (1994) 17953.

[31] J.P. Perdew, J.A. Chevary, S.H. Vosko, K.A. Jackson, M.R. Pederson, D.J. Singh, C. Fiolhais, Phys. Rev. B 46 (1994) 6671.

[32] H.J. Monkhorst, J.D. Pack, Phys. Rev. B 13 (1976) 5188.

[33] EDM 2.0.1; 〈www.numis.northwestern.edu/edm〉 using conditions for Hitachi H9000 microscope for an acceleration voltage of $300 \mathrm{kV}$, spherical aberration coefficient for the objective lens; $\mathrm{Cs}=1.1 \mathrm{~mm}$, the halfwidth of a Gaussian spread of focus due to chromatic aberration; DEL $=60 \AA$, and the semi-angle of incident beam convergence; $\mathrm{TH}=1.0 \mathrm{mrad}$.

[34] A. Loiseau, F. Willaime, N. Demoncy, G. Hug, H. Pascard, Phys. Rev. Lett. 76 (1996) 4737.

[35] Simone S. Alexandre, Mario S.C. Mazzoni, Helio Chacham, Appl. Phys. Lett. 75 (1999) 61.

[36] F. Jensen, H. Toflund, Chem. Phys. Lett. 201 (1993) 89.

[37] X. Blase, A. De Vita, J.-C. Charlier, R. Car, Phys. Rev. Lett. 80 (1998) 1666.

[38] T.M. Schmidt, R.J. Baierle, P. Piquini, A. Fazzio, Phys. Rev. B 67 (2003) 113407.

[39] Simone S. Alexandre, Helio Chacham, R.W. Nunes, Phys. Rev. B 63 (1999) 045402.

[40] P.W. Fowler, K.M. Rogers, G. Seifert, M. Terrones, H. Terrones, Chem. Phys. Lett. 299 (1999) 359.

[41] E. Bengu, O. Gulseren, Unpublished work on 8-fold and 10-fold BN rings; simulated images from BN basal planes with 8 -fold or 10 -fold defects were found to bear resemblance to structures pointed out in figure 3 (a). However, total energy values calculated for 8 -fold and 10-fold BN rings using the same procedure detailed in section 3 of this paper, were significantly higher than those calculated for 4-fold and 5-fold BN rings. 